http://dx.doi.org/10.18778/2196-8403.2015.14

MARTA WIMMER

\title{
Von brüchigen Familien und unglücklichen Singles - zu einem aktuellen Thema deutschsprachiger Gegenwartsromane
}

Artykuł stanowi próbę analizy najnowszych powieści literatury niemieckojęzycznej (Tommy Jaud, Angelika Reitzer i Marlene Streeruwitz) w kontekście zmienionej panoramy relacji partnerskich XXI wieku, która może być traktowana jako wynik niezdolności do kompromisu generacji wychowanej w przekonaniu o nienaruszalności jej własnej indywidualności. Ponadto poruszone zostało pytanie, jakie modele związków i style życia zostały przedstawione w wybranych tekstach literackich oraz jakie mechanizmy są za te zmiany odpowiedzialne.

Der Beitrag stellt einen Versuch dar, neueste deutschsprachige Romanwerke (Tommy Jaud, Angelika Reitzer und Marlene Streeruwitz) im Kontext des veränderten Partnerschaftspanoramas im 21. Jahrhundert zu analysieren. Dieses ist auf die Kompromissverweigerung einer Generation, die in der Überzeugung von der Unantastbarkeit ihrer eigenen Individualität sozialisiert wurde, zurückzuführen. Darüber hinaus wird auf die Frage eingegangen, welche Lebens- und Beziehungsmodelle in den ausgewählten Romanen in Erscheinung treten und welche Mechanismen diesen Veränderungen zugrunde liegen?

The article is an attempt to analyse the newest Germanophone novels (Tommy Jaud, Angelika Reitzer and Marlene Streeruwitz) in the context of changed patterns of partnership in the 21st century, which can be associated with a refusal to compromise by a generation brought upin the conviction of inviolability of their own individuality. Furthermore, the question has been raised what kind of partnerships and ways of living appear in the selected novels and which mechanisms are responsible for those changes.

Die Unbeständigkeit der heutigen Welt und die aus gesellschaftlichen Transformationen resultierende Labilität des Daseins tragen zu einer veränderten Partnerschaftskultur bei. Der stets steigende Wohlstand, der soziale Aufstieg 
von Frauen, die wirtschaftliche Unabhängigkeit sowie die Kommunikationsrevolution gehören laut ERIC KLINENBERG $(2012)^{1}$ zu den entscheidenden Faktoren, die für diese Veränderung verantwortlich sind. Tradierte Beziehungsmuster und alte Rollenvorstellungen lösen sich zunehmend auf, die Anzahl alleinlebender Menschen nimmt dagegen explosionsartig zu, gleichzeitig verliert die Institution der Ehe in ihrer herkömmlichen Auffassung an Bedeutung. Die Formen des menschlichen Zusammenlebens sind dafür in den letzten Jahrzehnten vielfältiger geworden. Das Singledasein sowie alternative Familienmodelle stellen heutzutage keine Ausnahme mehr dar, sondern sind zu einem festen Bestandteil der Wirklichkeit geworden. Literatur als kulturelles und historisches Produkt, „dessen Entstehung stets in einem spezifischen zeitlichen sowie gesellschaftlichen Kontext eingebunden ist" und die gleichzeitig als eine Form der „kulturellen Selbstwahrnehmung und Selbstthematisierung“ (GANSEL / HERMANN 2013:13) aufzufassen ist, setzt sich auch mit der Frage nach der Belastbarkeit von Beziehungen in einer brüchigen Welt auseinander. Der Fokus dieses Artikels wird auf aktuelle deutschsprachige Romanwerke (von Tommy Jaud, Angelika Reitzer und Marlene Streeruwitz), die neue Lebensformen und Partnerschaftsmodelle sowie deren Splitterformen thematisieren, gerichtet. Die Auswahl der Texte mag zwar im Hinblick auf ihre ästhetische Qualität recht willkürlich erscheinen, inhaltlich sind jedoch alle drei Texte literarische Repräsentationen, in denen die gesellschaftliche Realität in den Blick genommen wird. Von dieser Prämisse ausgehend soll danach gefragt werden, welche Lebensformen und Beziehungsmodelle in den ausgewählten Romanen in Erscheinung treten und welche Mechanismen diesen Veränderungen zugrunde liegen.

Der amerikanische Soziologe Erik Klinenberg, der an der New York University tätig ist, interviewte mit seinem Team in den letzten sieben Jahren über 300 alleinlebende Menschen und stellte dabei fest, dass die Idee von Singles als vereinsamte, frustrierte Menschen mit ausgeprägtem Neidkomplex der Vergangenheit angehört. In seinem 2012 erschienenem Buch Going Solo. The Extraordinary Rise and Surprising Appeal of Living Alone nennt er auch Gründe, die für die Verstärkung des Trends zum Alleinleben in der westlichen Welt verantwortlich sind. Siehe dazu auch das Interview in Die Presse: http://diepresse.com/home/leben/mode/749345/Vielleicht-der-groesste-soziale-Wandel-der-letzten-60Jahre?from=suche.intern.portal (28.06.2013). 


\section{Familie in Auflösung}

Bis Mitte des 20. Jahrhunderts blieb die Monopolstellung der Familie als Versorgungsinstitution, welche Funktionen wie Produktion, Konsumption, Sozialisation sowie Alters- und Gesundheitsvorsorge innehatte, weitgehend ungefährdet (vgl. PEUCKERT 2012:13). Seit den 70er Jahren wird in der deutschen Familiensoziologie die These von der Deinsitutionalisierung, die auch als Krise der Ehe und Familie wahrgenommen wird, vertreten. Diese besagt, dass sowohl die Ehe als auch die Familie an Plausibilität verloren hätten und dass sie einem Bedeutungswandel ausgesetzt seien (vgl. TYRELL 1979:61).

Die Ehe sei zu einer kündbaren Beziehung geworden, der institutionelle Verweisungszusammenhang zwischen Liebe, Ehe, Sexualität und dauerhaftem Zusammenleben habe sich gelockert, die Eltern-Kind-Beziehung sei keine Sonderbeziehung mehr [...], die Zugehörigkeit zu einer Familie erfolge nicht mehr nur aufgrund von Eheschließung und Filiation. (WAGNER / Franzmann / StAuder 2001:55)

Michael Wagner, Gabriele Franzmann und Johannes Stauder weisen darauf hin, dass,

[während] sich die Deinstitutionalisierungsthese auf private Lebensbereiche bezieht, [...] die Individualisierungsthese auch als Annahme über eine größere Optionsvielfalt bei der Wahl zwischen Beruf und Familie interpretiert [wurde]. Demnach ist ein zentrales Merkmal der Moderne die Freisetzung der Individuen aus den traditionell gewachsenen lokalen, familialen, ständischen und religiösen Bindungen. (WAGNER / FRANZMANN / STAUDER 2001:56)

In psychoanalytischer Auffassung gilt die Kernfamilie als Ort, „an dem das Selbst entsteht, der Ort also, an dem und von dem aus die Erzählung und Geschichte des Selbst ihren Anfang nimmt.“ (ILLOUz 2012:17) Gleichzeitig gehört sie zu jenen biographischen Ereignissen, die „symbolisch das ganze Leben begleit[en] und auf unverwechselbare Weise die eigene Individualität ausdrück[en].“ (ILLOUZ 2012:17) Die angesprochenen Veränderungen hinsichtlich der Stellung der Familie sowie die zunehmende Individualisierung gehören zu jenen Gegenwartsphänomenen, auf die die moderne Literatur reagiert und diese zugleich als Rahmenbedingungen des Schreibens integriert bzw. (kritisch) reflektiert. In allen drei hier angeführten Beispielen kann man von literarischen Repräsentationen gesellschaftlicher Realität ausgehen. In diesem Fall kann die These, dass Literatur als Seismograph gesellschaftlicher Veränderungen und Zustände angesehen werden könnte, erneut bestätigt werden.

In unter uns, dem zweiten Roman der österreichischen Autorin Angelika Reitzer, wird das Augenmerk auf das Bröckeln und Bröseln diverser Bindungen 
gerichtet. Das darin geschilderte Beziehungsgeflecht ist eher ein fragiles, was bereits dem Titel zu entnehmen ist. Dessen Kleinschreibung deutet nicht nur auf die offene Romankonstruktion hin, sondern verweist zugleich auf lose Beziehungen, die mithilfe der Figuren anschaulich gemacht werden:

Man kennt sich untereinander, zumindest von früher, zumindest vom Sehen: lauter Paare, Expaare, Derzeitsingles um die 40, die sich viel jünger fühlen, obwohl ihre Kinder schon recht groß sind, vielleicht auch, weil die meisten ihr Studentenleben weiterführen - prekäre Existenzen im Präkariat. (STRIGL 2010:19)

Der 2010 erschienene Roman thematisiert, wie sich verschiedene Arten von Bindungen allmählich auflösen, was als ein Zeichen der sich verändernden Welt gedeutet werden kann. Die um den eigentlichen Fixpunkt des Romans - Clarissa - kreisenden Figuren bilden einen hippen Freundeskreis kreativer Vierzigjähriger, die sich eher wie Anfang dreißig fühlen und gerne Mitte zwanzig wären (vgl. REITZER 2010:173). Die Autorin führt jedoch mithilfe ihrer Hauptfigur vor Augen, dass hinter diesem scheinbar perfekten Lifestyle nur noch Leere anzutreffen ist. Die Unzufriedenheit der ,Generation Projektarbeit', deren Gründe teilweise im beruflichen Bereich liegen, überrascht keineswegs. In einem Interview schildert die Autorin ihr Anliegen folgendermaßen:

Ich wollte die Schwierigkeit zeigen, ein klassisches Beziehungs- und Familienleben zu führen, wenn die konstituierenden Eckpfeiler einbrechen, beziehungsweise wenn es sie nicht mehr gibt. Sprich: Egal, welche Ausbildung du vorzuweisen hast, eine Anstellung, die ebenso fordert wie fördert, die gut (genug) belohnt ist, in der es Aufstiegschancen oder positive Ausstiegsszenarien gibt, ist keine, gar keine Selbstverständlichkeit mehr. Patchworksituationen in Familien- und Beziehungsgeflechten dagegen sind Normalität, die im Alltag bewältigt werden muss. Gleichzeitig verfolgen uns tradierte Familien-, Arbeits- und Lebensbilder, die mit unserer Realität - zumindest mit der eines Großteils von uns - kaum noch etwas zu tun haben. ${ }^{2}$

Der Roman beginnt mit einem Familienfest der anderen Art. Die Eltern von Clarissa, der eigentlichen Protagonistin des Romans, verabschieden sich nicht nur von ihrem beruflichen Leben, sondern auch (oder vor allem) von ihrer Familie und ihrer Elternrolle. Es ist nicht nur eine Wiedersehensfeier, sondern auch eine Abschiedsfeier: Clarissas Eltern brechen aus ihrem bisherigen Leben und gleichzeitig aus dem Leben ihrer Kinder aus, „sie haben sich ja bereits verabschiedet aus diesem Verband, sie haben sich distanziert und wollen, dass sie alle mit ihnen die Gläser darauf erheben.“ (REITZER 2010:29f.) Wie es

2 Reitzer, ANGEliKA im Interview mit Bettina Führer: http://www.buecher.at/show_content2.php?s2id=452 (25.06.2013). 
scheint, macht der Drang zur Selbstverwirklichung des modernen Menschen nicht einmal vor der älteren Generation halt.

Der Ausstieg von Clarissas Eltern ist präzise geplant [...]. Ihr Gasthof ist seit über einem Jahr verpachtet, nun haben sie alles verkauft. Wohin sie gehen werden, wollen sie nicht verraten, weil sie zu dem Zeitpunkt noch nicht sicher sind, ob sie überhaupt noch Kontakt zu irgendjemandem haben wollen. (REITZER 2010:30)

Wirklich erschüttert über die Entscheidung des Ehepaares Stadler scheint aber niemand zu sein. Nichtdestotrotz hinterlässt diese tiefgreifenden Spuren, vor allem was das Leben ihrer erwachsenen Tochter Clarissa anbelangt. Die innere Zerrissenheit und Leere, die sich in ihrem Leben breit machen, scheinen unüberwindbar zu sein. Da die Familie ihrer herkömmlichen Funktion beraubt wurde, verliert diese für die Protagonistin an Bedeutung. Bewusst bricht sie den Kontakt zu ihrer Schwester, die eine berühmte Schriftstellerin ist, ab. Auf die Frage, ob sie mit der Autorin Stadler verwandt ist, weil sie den gleichen Nachnamen trägt, antwortet sie mit einem dezidierten Nein und fügt hinzu: „ich habe keine Schwester, sagte ich da, und: Autorin, die so heißt wie ich, kenne ich keine.“ (ReITZER 2010:142) Infolge der Familienauflösung beginnt Clarissa auf ihre Kindheit, die auf Videokassetten verewigt wurde, zurückzublicken:

Ein Land, in dem ich niemals gewesen bin, in dem wir nie zusammen gewohnt haben. Alles ist dokumentiert, chronologisch geordnet, das Archiv meiner Kindheit in der großen Truhe in der Stube. [...] Auf manchen Filmen war vielleicht eine fröhliche, leicht disparate Familie zu sehen, die in einem Gastgarten probierte, wie es war, wenn man zusammengehörte. Es gab Aufnahmen von meinem sechsten Geburtstag, der wie alle Geburtstage mit der ganzen Familie gefeiert wurde, außerdem mit Kindern aus der Nachbarschaft. [...] Aber was sich auf den Filmen nicht fand, davon hatten die Eltern keine Vorstellung. Früher dachte ich immer, sie wollten das alles nicht sehen, es hätte sie nur traurig gemacht, dass sie immer Geld verdienen mussten, während wir mit unserem Onkel und seiner Frau auf Almen wanderten, irgendwo übernachteten oder an Bodenseen zelteten. Was konnten sie davon gewusst haben? Es wird sie nicht interessiert haben, warum auch? (REITZER 2010:10f.)

Die Erinnerungen täuschen jedoch nicht über die Wirklichkeit und die Tatsache, dass die Familie nicht mehr besteht, hinweg. Clarissas Kindheit gehörte zwar nicht zu den glücklichsten Etappen ihres Lebens, der Schein blieb jedoch aufrechterhalten. Aus einigen Rückblenden erfährt der Leser, dass die Eltern der Protagonistin trotz der physischen Präsenz in ihrer Kindheit immer abwesend waren. In einem gewissen Sinne wiederholt Clarissa das gleiche Muster: Zuerst ist sie eine fleißige und erfolgreiche Arbeitsbiene, steigt dann aber aus 
oder, besser gesagt, scheitert an ihrem Perfektionismus und ihrer Dünnhäutigkeit und erlebt einen Absturz. Das Leben über ihre Verhältnisse, Schulden und die Arbeitslosigkeit haben zur Folge, dass sie als Untermieterin im Haus (genauer gesagt im Keller) ihrer Freunde wohnt und nicht einmal mehr im Besitz einer Bankkarte ist. Die Perspektivlosigkeit der Figur erreicht in diesem Moment ihren Höhepunkt, was vor allem an ihrer Wohnsituation manifest wird. Wie Daniela Strigl treffend bemerkt, kann man diesen Roman auch räumlich verstehen: „Clarissa ist die Souterrainexistenz, die den dräuenden Untergrund der glücklichen Familie bildet, an die niemand so richtig glaubt.“ (STRIGL 2010:19) Die Familie, bei der Clarissa im Untergeschoß wohnt, erscheint auf den ersten Blick intakt. Aus Clarissas Perspektive wird das wie folgt beschrieben:

Manchmal war es so: der Tag hat gerade begonnen, die Mädchen schwirren gut gelaunt durch die Zimmer. Als wäre die Welt nur für sie da. Sie kennen nicht viel, sie kennen ja nur das kleine Glück ihrer intakten Familie. (REITZER 2010:61)

Das Familienglück der anderen wird für die Figur zunehmend unerträglich, die Lebensentwürfe dagegen kaum nachvollziehbar. An einer anderen Stelle liest man:

Als ich bei Tobias und Klara einzog, erwarteten die bereits ihr zweites Kind. Und freuten sich. Tobias stammte schließlich aus einer derart intakten Familie, dass es mir nur logisch vorkam oder natürlich, dass er das jetzt fortsetzen wollte. (REITZER 2010:150)

Klara und Tobias sind das einzige Paar im ganzen Roman, das ein traditionelles Familienmodell verkörpert, doch auch dieses beginnt allmählich zu bröckeln. Die traditionelle Familienauffassung, wonach der Mann berufstätig ist, die Frau sich hingegen um den Haushalt kümmert, scheint ausgedient zu haben. Die auf sich allein gestellte Frau, die den Anforderungen als Mutter nicht gewachsen ist, „stellt sich Fragen nach dem Sinn des Lebens, nach den zwischenmenschlichen Beziehungen in einer stets herausfordernden modernen Gesellschaft.“ (KŁOPOTOWSKA 2013:118) Dabei überlegt Klara, ob es nicht ein Schwellenzustand zwischen Eintreffen und Noch-Nicht-Angekommen-Sein ist (vgl. KŁOPOTOWSKA 2013:118).

Eine Familie, eine Familie. Es ist für Klara nicht immer selbstverständlich gewesen, dass es das ist, was sie sich wünscht, was sie vom Leben erwartet. Was für eine unnütze Frage. Das Leben, was sonst! Als wäre sie in einer Art Zwischenzustand, als hätte sie etwas zu überdauern, und dann. Und dann. (REITZER 2010:75f.) Familie bedeutet für Klara etwas Fesselndes, etwas, das sie in ihrem Handeln und Denken einschränkt und nur noch Angst hervorruft: 
Aber wenn sie ihre Kinder atmen hört, überkommt sie manchmal diese Einsamkeit und Angst, die sie erst kennt, seitdem sie Mutter ist. Eine Form des Alleinseins, die sie früher nie gespürt hat und die sie sich gar nicht vorstellen hätte können im Zusammenhang mit einem Mann, der ihr Mann ist, stark und klug und an- und abwesend, und mit den Kindern, ihren Kindern. [...] Diese Ahnung oder Furcht, sich aufzulösen oder zu verschwinden, vielleicht unter all dem, was ihr Leben ist. Vielleicht ist es der Schrecken, nicht mehr da sein zu können für ihre Familie. (REITZER 2010:107f.)

Die Mutterrolle ist für Klara eine widersprüchliche Erfahrung, da sie sich als Frau verwirklichen möchte, sich jedoch zugleich in einen gesellschaftlichen Rahmen gedrängt fühlt. Den Aufgaben, die man ihr als Frau stellt, ist sie nicht ganz gewachsen, diese lösen bei ihr ein Gefühl des Begrenztseins, der Unsicherheit und der Vereinsamung aus. Reitzer vermittelt ein lebendiges Bild von Paaren, die sich auseinandergelebt haben. Beziehungen, auf die das Augenmerk des Romans gerichtet wird, funktionieren „nur noch als etablierte Distanzverhältnisse“. ${ }^{3}$ Die Autorin schafft mit ihrem Roman ein komplexes Gesellschaftspanorama, erzählt wird vor allem von der Generation, die über dreißig Jahre alt ist und im beruflichen Bereich schon alles ausprobierte, jedoch auch in diesem Alter noch nicht angekommen ist. Das Unverbindliche, unverbindliche Meetings und unverbindliche Beziehungen scheinen symptomatisch für die ganze Generation zu sein. Das, was eindeutig fehlt, ist ein Gemeinschafts- und Zugehörigkeitsgefühl, was auch folgende Passage augenscheinlich macht: „Aber dieses Wir gibt es nicht, gab es nie“ (REITZER 2010:266). Hier legt die Literatur den Finger auf offene Wunden, indem sie die brüchig gewordenen Bindungen reflektiert. Die geschilderten zwischenmenschlichen Beziehungen erwecken ganz und gar nicht den Anschein von Stabilität, man lebt zwar zusammen, doch mit einem intakten Gemeinschaftsleben hat das kaum etwas zu tun. Der literarische Text arbeitet ,an den zentralen Bruchlinien unserer Gesellschaft, denn für jede aufgebrochene Struktur, auch die familiäre [...] müssen Alternativen gefunden werden.“ (POLT-HeINZL 2013:19f.) Zwar repräsentieren die Protagonistinnen verschiedene Lebensentwürfe, doch das, was sie gemeinsam haben, ist die Tatsache, dass keiner von diesen Lebensstilen als ein Garant von Stabilität gesehen werden kann, worauf auch eine gewisse Ähnlichkeit im Hinblick auf die Namen der weiblichen Figuren (Klara, Klärchen, Clarissa) hindeutet. Die mangelnde Sicherheit innerhalb zwischenmenschlicher Bindungen bzw. das Fehlen dieser findet ihre Widerspiegelung

3 Pohl, IsABELla: Gemeinsam sein und doch getrennt: http://derstandard.at/ 1282273499151/Gemeinsam-sein-und-doch-getrennt-leben (29.06.2013). 
im Erzählduktus der Autorin. Der an manchen Stellen ein wenig nervös wirkende Erzählstil illustriert treffend die mentale Grundverfassung des modernen Menschen.

\section{Single als Lebensmodell?}

Die Singles stellen eine historisch recht junge soziale Gruppe dar, die die Öffentlichkeit zu polarisieren scheint. Die Extensität der öffentlichen Diskussion lässt darauf schließen, dass das Singledasein mehr als eine reine Privatangelegenheit ist, was auch das Interesse der Wissenschaft für dieses Phänomen bestätigt. 1995 verkündete Stefan Hradil, Sozialwissenschaftler und Singleforscher, Singles seien aufgrund der Verbreitung dieses Phänomens sowie des Interesses der Presse an diesem zu einer öffentlichen Angelegenheit geworden (vgl. HRADIL 1995:V). Sieben Jahre später konstatiert die Sozialpsychologin Beate Küpper, dass die Wahrnehmung des Singledaseins durch die mediale Darstellung dermaßen beeinflusst werde, dass sich eine Verflechtung zwischen medialem Bild und Wirklichkeit feststellen lasse.

Das heißt für den Single: Ist er deckungsgleich mit seinem Bild in den Medien, kann nicht beurteilt werden, was zuerst da war. Ist er nicht deckungsgleich, kann zumindest die Möglichkeit in Betracht gezogen werden, daß er einfach so lebt, wie er lebt. Zumindest scheint es für eine Gruppe, die so sehr im Zentrum der öffentlichen Aufmerksamkeit steht wie die Singles schwierig zu sein, sich dem medialen Bild zu entziehen. (KüPPER 200:63f.)

Einen wesentlichen Einfluss auf das medial vermittelte Bild des Singledaseins haben seine fiktionalen Umsetzungen und es lässt sich um die Wende zum 21. Jahrhundert im Kino und Fernsehen sowie auf dem Buchmarkt eine extreme Häufung von Publikationen beobachten, die das Singledasein thematisieren. Das Interesse an der zahlenmäßig recht kleinen Gruppe führen Stefan Hradil und Ulrich Beck auf die Tatsache zurück, dass die Singles eine Art soziale Vorreiterrolle innehaben. Im Vergleich zum Rest der Bevölkerung streben die Singles deutlich öfter nach ,neuen“ Werten wie Entscheidungsfreiheit oder Selbstverwirklichung und wesentlich seltener nach traditionellen Werten wie Pflichterfüllung, Ordnung oder Sicherheit. Gleichzeitig lassen sich an der Lebensweise dieser sozialen Gruppe sowohl Auswirkungen der Frauenemanzipation als auch gewisse Veränderungen auf dem Arbeitsmarkt ablesen (vgl. CLAus 2012:13f.). ${ }^{4}$ Geht man von der Feststellung aus, dass wir es in der fik-

Vgl. dazu auch BECK (1986:199f.) und BECK / BECK-GERNSHEIM (1990:78-91). 
tionalen Darstellung mit einem ähnlichen Bild des Singles zu tun haben, könnte man annehmen, „dass sich anhand der Figur des Singles moderne gesellschaftliche Problem- und Bewusstseinslagen besonders konzentriert darstellen und diskursiveren lassen.“ (CLAus 2012:14f.) Da die erwähnten Soziologen ihre Thesen noch lange vor der Hochkonjunktur der Singlenarrative aufstellten, wäre zu fragen, inwiefern diese Bilder miteinander kompatibel sind oder in welchem Ausmaß sie sich gegenseitig beeinflussen?

In der fiktionalen Darstellung treten Singles gehäuft erst ab Mitte der 1990er Jahre auf. Es soll an dieser Stelle jedoch angemerkt werden, dass in diesen Texten überwiegend Frauen im Mittelpunkt der Handlung stehen. Eine davon ist Jessica Somner, ${ }^{5}$ die Protagonistin des Romans Jessica, 30. der österreichischen Autorin Marlene Streeruwitz, „die in wesentlichen Zügen ganz nach dem genreüblichen Muster der Singleromane gestaltet ist.“ (CLAUs 2012:143) Solche ,Illustriertennamen' wie Jessica oder Lisa (so der Name der Titelfigur des 1997 erschienenen Romansammelbandes Lisa's Liebe; STREERUWITZ 1997) „reserviert die Autorin für jene Papierfiguren, welche demonstrativ an der Grenze zum Trivialen siedeln.“ (ZINTZEN 2005:68) Der Text nimmt in vielerlei Hinsicht eine Sonderstellung in dem Gesamtœuvre der Schriftstellerin ein, denn nicht nur gehört die Hauptfigur einer anderen Generation an als die Heldinnen ihrer bisherigen Texte, sondern ,auch weicht das typografische Erscheinungsbild aufgrund der veränderten Syntax auffällig von demjenigen der vorausgegangenen Romane ab“ (CLAUS 2012:132). Streeruwitz übernimmt zwar diverse für den Singleroman der modernen Unterhaltungsliteratur typische Klischees, doch indem sie diese in einen anderen genreüblichen Kontext einbettet, verleiht sie ihnen eine andere Aussagepotenz (vgl. Claus 2012:134). Ihren fünften Roman widmet Streeruwitz den „Wesens-, Lebens- und Leidensarten weiblicher Thirtysomethings“ (ZINTZEN 2005:68). Die Zahl ,30‘, die bereits im Titel des Romans vorkommt, gewinnt eine besondere Bedeutung. Es handelt sich nicht nur um eine schlichte Verortung der Figur im Hinblick auf

Der Name Jessica Somner erinnert sehr stark an die Bravo-Expertin Dr. Sommer, die Jugendliche in Fragen der Liebe und Sexualität beriet. Der erste Dr. Sommer war der Psychotherapeut Martin Goldstein, in den letzten Jahren leitete Jutta Stiehler die Rubrik in der Jugendzeitschrift, die im Jahre 1956 gegründet wurde und zu den bekanntesten deutschen Jugendmagazinen gehört. Inzwischen werden die Fragen der Jugendlichen von einer Erzieherin beantwortet. Vgl. dazu BALLER, SuSANNE (2015): „Was in der ,Bravo“ geschieht, ist sexistisch und frauenfeindlich “: http://www.stern.de/familie/kinder/-sexistisch-und-frauenfeindlich---dr-sommer-geht-auf--bravo--los-6357054.html (18.09.2016). 
ihr Alter, die Zahl markiert vielmehr „ein gruppenspezifisches Lebensgefühl und ist damit zugleich individuelles Identitäts- und übergeordnetes Generationsmerkmal.“ (ClAUs 2012:133) Der Zusatz der Altersangabe ist somit als ein Verweis auf die „Beispielhaftigkeit des Einzelschicksals“ (CLAUs 2012:133) aufzufassen. Jessica ist eine dreißigjährige, aus der Mittelschicht stammende Westeuropäerin, besitzt einen geisteswissenschaftlichen Universitätsabschluss, hat im Fach Kulturwissenschaft promoviert und arbeitet als Journalistin bei einer Frauenzeitschrift, ist also wie im Genre Single-Roman üblich, in der Medienbranche tätig. Streeruwitz' Roman weist aber auch signifikante Brüche in Bezug auf die genreübliche Figurenkonzeption auf, bei ihr gewinnen die Fragen der Lebensunterhaltsfinanzierung und der Karriereplanung erhöhte Bedeutung, worauf Britta Claus in ihrer Monographie Kein Leben zu zweit hinweist (vgl. CLAus 2012:143). Der Arbeitsplatz wird einerseits zur Grundlage einer gesicherten Lebenssituation und somit unabhängiger Lebensführung, andererseits wird er aber als Ort des Zwangs und der Anpassung gesehen, der die Protagonistin daran hindert, sich selbst zu verwirklichen:

[D]ie Tanja ist intelligent, sie ist die Intelligenteste da, überhaupt, und ich verachte sie doch nicht, weil sie für so ein Massenblatt arbeitet, es ist natürlich schade um ihre Ausbildung, aber vielleicht projiziert sie das auf mich, auf uns, wir waren da ehrgeizig, ehrgeiziger, und dass sie als Psychologin da, findet sie das einen Abstieg, findet sie das einen Verrat, weil sie ja eigentlich etwas Therapeutisches machen wollte und da noch lange kein Geld verdient hätte, ich verstehe das doch, dass man es jetzt ordentlich haben will, wir haben auch ein Recht auf einen Lebensstil, und dann muss man halt solche Sachen machen, ich hätte ja auch nicht gedacht, dass ich einmal Sexratgeber schreiben werde und Foucault auch keine Rolle mehr spielt. (STREERUWITZ 2004:84)

Der Arbeitsplatz, der einen wesentlichen Bestandteil des Lebens und Denkens der Hauptprotagonistin ausmacht, wird von ihr zudem als ein Ort des „Kampf[es] mit den bösen Kräften“ (STREERUWITZ 2004:86) betrachtet. Aus diesem Kampf geht Jessica Somner trotz ihres Karrierestrebens jedoch als Verliererin hervor, als eine, die ihre Ideale verraten und sich dem System angepasst hat. Fehlende Festigkeit lässt sich allerdings nicht nur im beruflichen, sondern auch im partnerschaftlichen Bereich konstatieren. Das Familienleben, das für die ältere Generation eine Selbstverständlichkeit war, scheint für die „Post-Brigitte-Generation“6 (MENSING 2004:38) eine Wunschvorstellung, jedoch keine Realität darzustellen:

$6 \quad$ Mit der ,Brigitte-Generation“ sind Frauen gemeint, die mit der Zeitschrift - zu ihrer Blütezeit (1980er) - aufgewachsen sind, ihr politisches Bewusstsein erlangten sowie ihr berufliches Leben begannen. Die typische Vertreterin dieser Generation 
[D]ie Mama hat heute nur, was sie schon mit 30 gehabt hat, den Job, das Kind, nur der Mann hat gewechselt, und ich habe keinen Job, kein Kind und die Männer sind Wechselbälger, seit dem Alfred nur noch irgendwelche und die Beziehungsproblematik und keine Aussicht auf eine Festigkeit (STREERUwITZ 2004:27).

Obwohl die Protagonistin überzeugt ist, dass es „keine Zeit für Singles ist, obwohl niemand eine Familie ernähren könnte“ (STREERUWITZ 2004:53), führt sie diesen Mangel an gefestigten Strukturen deutlich weniger auf gesellschaftliche Entwicklungen zurück, die sowieso außerhalb ihres Einflussbereiches liegen. Vielmehr macht sie hierfür individuelle lebensgeschichtliche Erfahrungen verantwortlich. Die Schuld für ihre Bindungsangst bzw. -unfähigkeit gibt sie zunächst dem abwesenden Vater (vgl. STREERUWITz 2004:64). Letztlich scheint jedoch die Beziehung mit ihrem Exfreund Alfred ausschlaggebend für ihre jetzige Lebenssituation zu sein:

[V]om Alfred weiß ich auch nur mehr, wie er auf diesen Brücken gestanden ist und wie wir ins Wasser schauten, dieses schwarze Wasser, [...] und wie ich ein einziger Klumpen bin, ein einziger Klumpen, der wehtut, ein Schmerzklumpen, [...], und bei jedem Satz von ihm wird der Klumpen fester, bis man gar nicht mehr gehen kann und als Salzsäule dasteht, und natürlich in Venedig, wo sonst erklärt einem ein Mann, dass er nicht mehr schlafen will mit einem, und dass sein Recht ist, dass niemand ein Recht darauf hat, darauf, dass man mit der anderen Person ins Bett geht, nur weil sie einen liebt und weil man das nun seit 2 Jahren gemacht hat, und wo sonst täte das noch so weh. (STREERUWITZ 2004:215f.)

An einer anderen Stelle liest man:

[W]ahrscheinlich ist Venedig der Ort, an dem man das sowieso sagt, wahrscheinlich sind da alle Liebeserklärungen ohnehin nur diese Sätze, das Ende ist einem ja ohnehin klar, und wenigstens habe ich nicht zugegeben, dass ich schon mit 2 anderen Männern in Venedig war, und dass ich mit jedem das Gleiche gemacht habe und bevor er mir diese Sätze gesagt hat, [...] [wäre] es [...] besser gewesen, er hätte mich betrogen und hätte mich angelogen, dann gäbe es wenigstens Venedig noch so, wie es das immer gegeben hat. (STREERUWITZ 2004:216)

Der Ort des Geschehens, Venedig, verkörpert für Jessica „zunächst das romantische Potenzial der Liebe, das sie für sich nutzen kann - und auch bereits mit anderen Partnern genutzt hat [...], dessen Wirkungsmacht aber dann für sie durch Alfreds Zurückweisung gestört wird“ (ClAus 2012:145). Die Protagonistin ist keineswegs nur Opfer männlicher Gefühllosigkeit, ihr Beziehungsverhalten ist alles andere als altruistisch, was ihre Selbstaussage: „[I]ch hole

ist eine berufstätige, verheiratete Frau mit Kindern. Die Protagonistin des Romans ist dagegen eine typische Vertreterin der ,Post-Brigitte-Generation’: berufstätig, kinderlos, Single. 
mir ja, was ich will“ (STREERUWITZ 2004:6) bestätigt. Sie weiß das romantische Potenzial der Stadt Venedig auszunutzen und wiederholt diese Inszenierung, deren Ende abzusehen ist, bei mehreren Männern. Nur bei Alfred versagt diese Strategie. Der Verlust des „Urvertrauen[s]“ (STREERUWITZ 2004: 130), von dem die Protagonistin spricht und das als eine unerlässliche Voraussetzung einer jeden Liebesbeziehung gilt, sowie die damit zusammenhängende Angst, ein weiteres Mal abgewiesen zu werden, tragen dazu bei, dass die Protagonistin nicht in der Lage ist, sich ohne Vorbehalte auf einen Mann einzulassen. Obwohl der Wunsch danach durchaus vorhanden ist:

[I]ch möchte es auch gemütlich haben, und ich möchte es innig, mich in jemanden einwickeln, sich ineinander gemeinsam verbergen, und ein Schutz, ich möchte eine problemlose Rückkehr in die Kindheit und ich möchte jedes Mal die Rückfahrkarte haben und nicht da zurückgelassen werden, wenn ich einmal das Urvertrauen gefasst habe. (STREERUWITZ 2004:130)

Im Laufe des Romans gewinnt das Konzept von Stabilität und Sicherheit immer mehr an Bedeutung. Die Fixierung auf das Erreichen sexueller Erfüllung, die noch im ersten Kapitel thematisiert wurde, gerät allmählich in den Hintergrund:

[Z]wei Personen auf der Suche nach der sexuellen Erfüllung und wie schwierig das ist und was für komische Leute man dabei trifft und wie man von diesen Partnern abhängig ist, dass man seine sexuelle Erfüllung eben nicht alleine spielen kann, da braucht man immer andere dazu und was man tun könnte, damit die funktionieren, und wenn man sich verliebt hat, dann geht das alles schon wieder nicht, dann ist das alles schon wieder etwas anderes und man verliert seine Wünsche wieder aus den Augen [...] und man möchte doch nur einen erwachsenen Mann finden. (STREERUWITZ 2004:125f.)

Gegen Ende des Romans taucht in Jessicas Leben der Arzt Harti auf, der eine Kontrastfigur zu dem ,Alphamännchen` und Geliebten der Protagonistin Gerhard Hollitzer darstellt. Harti, eine fast ausschließlich positiv gezeichnete Nebenfigur, verkörpert nahezu alle Eigenschaften, die Jessica von einem potenziellen Lebenspartner erwartet. Obwohl die sich anbahnende Beziehung zu Harti zunächst auf etwas Festes hoffen lässt, wird dem Leser schnell klar, dass man eher ein erneutes Scheitern als ein Happy End erwarten wird.

Nicht nur die Möglichkeit, eine Bindung mit einem anderen Menschen einzugehen, wird von der Figur in Frage gestellt. Problematisch erscheint auch oder vor allem die Beziehung zu sich selbst, was sich in Jessicas Einstellung zu ihrem eigenen Körper oder, besser gesagt, in der Gegenüberstellung ,Kopf‘ vs. ,Körper‘ manifestiert. Die Figur erweist sich als eine höchst kopfgesteuerte 
Person, die sich aber damit abmüht, den Bedürfnissen ihres Körpers zu folgen (vgl. Claus 2012:146). Sie selbst formuliert das folgendermaßen:

[V]ielleicht muss ich da eine Schwelle überwinden, vielleicht muss ich meinen Körper da in etwas vorausschicken und dann nachkommen, mit dem Kopf, aber das hat eben nie funktioniert, das funktioniert nicht bei mir. (STREERUWITZ 2004:114)

Das Bild der Heldin ist durchaus klischeehaft, „[w]ahrscheinlich soll es eine Kritik an diesen von Frauenzeitschriften und amerikanischen Fernsehserien genormten Monster-Großstadt-Singlefrauen sein. “7 Das von Streeruwitz skizzierte Porträt vervielfältigt jedoch diese Klischees, anstatt sie abzubauen. Jessica joggt, weil sie in der Nacht ,gesündigt' d.h. einen ganzen Eisbecher gegessen hat, und überlegt dabei, ob die Fettsucht nicht doch „der Ausgleich für mangelnde Sexualität“ (STREERUWITZ 2004:6) ist. Die Strategie der Autorin, dem Leser einen Einblick in die Gedankenwelt der Protagonistin zu gewähren, macht anschaulich, dass sich die Gedanken der Figur im ständigen Spannungsfeld zwischen Intellektualität und Trivialitäten des Singlealltags befinden. Diese Ambivalenz wird besonders deutlich im Hinblick auf das Thema Sexualität, wobei die feministische Position auf die der Sex-and-the-City-Serie ${ }^{8}$ entnommenen Weisheiten bezüglich Weiblichkeit, Machtverhältnissen oder sexueller Befreiung stößt. Das Singledasein wird in diesem Roman auf die Spitze getrieben: Jessica ist ein Mensch ohne soziale Einbettung. Die Protagonistin hat keinen Partner, keine wahren Freunde oder Geschwister. Auch die Eltern, die ihre letzte soziale Bindung zu sein scheinen, nimmt die Protagonistin als solche nicht wahr. Sie existieren für sie nur noch als getrennte Einzelpersonen, da sie längst geschieden sind und der Vater mit einer Frau liiert ist, die nicht viel älter als Jessica ist.

Streeruwitz' Text, in dem die Autorin den Gedankenstrom einer autodiegetischen weiblichen Erzählinstanz entwirft, gehört zu jener Reihe von Romanexperimenten, die „das Konzept melancholischer Liebe neu erproben und ausbuchstabieren“, jedoch kein Ende dieser konstatieren. „Es bleibt konstant bei der dialektischen Inszenierung von empathischer Affirmation und kritischer

7 AdORJÁN, JoHAnNA: Sie und ihr Maple Walnut: http://www.faz.net/aktuell/feuilleton/buecher/rezensionen/belletristik/sie-und-ihr-maple-walnut-1163911.html (13.06.2013).

,Sex and The City“ ist eine erfolgreiche amerikanische Fernsehserie, die frei auf dem gleichnamigen Roman von Candance Buschnell basiert. Erzählt wird die Geschichte von vier in New York lebenden Freundinnen, die ihr (Liebes)Leben bis ins kleinste Detail miteinander besprechen. 
Analyse.“(METZ 2012:433) Das Experimentieren der Hauptfigur mit verschiedenen Partnerschaftsmodellen könnte auch im Kontext der Neudefinierung der Rollenverteilung gelesen werden (vgl. CLAus 2012:130).

Der fiktionale Singlediskurs ist größtenteils weiblich bestimmt. Schilderungen des Singledaseins aus der männlichen Perspektive bilden in der deutschsprachigen Gegenwartsliteratur eine Ausnahme. Britta Claus weist darauf hin, dass in den letzten fünfzehn bis zwanzig Jahren Zielgruppen, Verfasser und Hauptfiguren von Texten, die das Singledasein thematisieren, nahezu ausschließlich weiblich sind (vgl. Claus 2012:73). Den Befund, dass die weiblichen Singlefiguren in der deutschsprachigen Unterhaltungsliteratur der jüngeren Gegenwart überwiegen, führt die Autorin auf „zeitlich früher einsetzende soziokulturelle Entwicklungen [...], die in Zusammenhang mit der Frauenemanzipationsbewegung stehen“ (CLAUS 2012:74), zurück.

Zu den wenigen Ausnahmen, die das Singledasein aus männlicher Perspektive schildern, zählen Romane deutscher Autoren wie Tommy Jauds Vollidiot, Daniel Bielensteins Die Frau fürs Leben oder Björn Erik Sass’ Herrenbesuch. Der 2004 erschienene Roman Vollidiot, der eine Art männliches Pendant zu dem weiblich bestimmten Singleroman darstellt, stand monatelang auf den Beststellerlisten und wurde 2007 unter der Regie von Tobi Baumann verfilmt. ${ }^{9}$ Mit diesem Buch sowie mit dem 2006 erschienenem Roman Resturlaub hat der Autor „ein brachliegendes Genre neu belebt - den deutschen Männerroman“, so WOLFGANG HÖBEL (2006). „Da die genderspezifischen Veränderungen der Gesellschaft eine relativ neue Erscheinung sind, ist auch die Unsicherheit der Männer bezüglich ihrer Männlichkeit eine neue Erscheinung, ebenso wie der Männerroman, in dem diese Unsicherheit zum Thema gemacht wird.“ (KNAUP 2015:12) Die diagnostizierte Unsicherheit bzw. Gender-Krise, von der in diesem Zusammenhang häufig die Rede ist, ist überwiegend auf die Auflösung der traditionellen „Separation von gesellschaftlichen Männer- und Frauenbereichen“ (vgl. KNAUP 2015:12) in den modernen Gesellschaften zurückzuführen. Männerromane dieser Art werden zwar dem niederen Literaturniveau zugerechnet, dies ändert jedoch nichts an der Tatsache, dass sie ein interessanter Forschungsgegenstand sind, dem allerdings bisher - höchstwahrscheinlich wegen ihrer mangelnden ästhetischen Qualität - wenig Aufmerk-

$9 \quad$ Vgl. VolLidiOT, BRD 2007, Regie: Tobi Baumann, Drehbuch: Tommy Jaud und Christian Zübert, Produktion: Sven Burgemeister und Christoph Müller. 
samkeit geschenkt wurde. ${ }^{10}$ Die Wahl ausgerechnet dieses Textes für die Analyse lässt sich primär durch seinen thematischen Schwerpunkt begründen. Trotz der mehrfach erwähnten qualitativen Schwachstellen des Romans hat man es mit einem durchaus interessanten Phänomen der Gegenwartsliteratur, das im gewissen Sinne die gegenwärtige Problemlage thematisiert, zu tun.

Der Kölner Autor schildert unterhaltsam das vermeintliche Leid des Singledaseins, wobei er allerdings manchmal ins Niveaulose driftet. Der 29-jährige Protagonist Simon Peters, ein Telekom-Mitarbeiter, ist erneut Single und befindet sich gerade in der diesbezüglichen vierten Phase,

bestehend aus purer Verzweiflung in Verbindung mit einem stetig bröckelnden Selbstbewusstsein. Das Schlimme daran ist, dass es sich um einen Teufelskreis handelt: Je größer das Bedauern, desto geringer die Möglichkeiten, und je geringer die Möglichkeiten, desto größer das Bedauern. (JAUD 2012:29)

Seinen Gemütszustand macht er auch für seinen Misserfolg bei den Frauen verantwortlich, nichtsdestotrotz gibt er die Suche nach ,Miss Perfect' nicht auf. Dabei soll ihm ein Urlaub in einem Single-Club auf den Kanaren helfen. Weder in diesem Club-Urlaub noch im Kölner Nachtleben findet er die große Liebe und bei Ikea begegnet er lauter Pärchen, wodurch er schmerzhaft daran erinnert wird, dass er ein Versager ist:

[Es] gibt für einen Single nichts Ungeeigneteres, als einen verregneten Oktobersamstag bei Ikea zu verbringen. Das ist garantiert auch der Grund, warum man dort keine Singles sieht. [...] Nirgendwo auf der ganzen Welt wird einem Single sein ganz persönliches Scheitern konzentrierter und kaltblütiger vor Augen geführt als bei Ikea. Und zwar auf der ganzen Linie. Ikea, das ist kein Einrichtungshaus, das ist ein ausgeklügelter, skandinavischer Lehrpfad des Versagens, der durch das eigene Nichts direkt zu einer der dreißig gelb-blauen Kassen führt. (JAUD 2012:7f.)

Letztendlich verliebt sich der Protagonist Hals über Kopf in eine StarbucksThekenkraft namens Marcia, mit der er sich eine gemeinsame Zukunft vorstellen kann. Doch als er sie anspricht, fühlt er sich wie „wie ein Ossi beim ersten McDonalds-Besuch, drei Minuten nachdem die Mauer gefallen ist.“ (JAUD 2012:116) Schließlich gelingt es Simon jedoch, ein Treffen mit seiner Angebeteten zu arrangieren, er lädt sie zu einem Konzert der Hip-Hop-Band ,Die Fantastischen Vier ${ }^{`}$ ein, muss dann aber feststellen, dass die Frau seiner Träume nur eine in seiner Phantasie existierende Wunschvorstellung ist und

Eine Ausnahme stellt die 2015 erschienene Monographie Der Männerroman: Ein neues Genre der deutschsprachigen Gegenwartsliteratur von ANNA KATHARINA KNAUP dar. 
diese nicht der Realität entspricht. Die enttäuschte Hoffnung sowie sein zeitnaher Jobverlust haben zur Folge, dass sich Simon vom Rest der Welt abkapselt und in zeitweiliger Isolation lebt. An seinem dreißigsten Geburtstag wird ihm aber klar, dass er auf seine Freunde zählen kann. An diesem Abend beginnt er seine Lage (vor allem aber seine ehemalige Chefin) mit anderen Augen zu sehen und beschließt dem Unglück exakt an dem Ort, an dem es angefangen hat, ein Ende zu setzen. In einer Nacht-und-Nebel-Aktion verbrennt er den zuvor bei Ikea gekauften Jennylund-Single-Sessel vor dem schwedischen Kaufhaus. Mit diesem Ende schafft der Autor eine Klammer, die sowohl die Hauptfigur als auch den Leser zum Anfang zurückführt. Wie in den meisten SingleRomanen geht es auch bei Tommy Jaud um die Entwicklung des Hauptprotagonisten, auf seinen fünf Etappen der Trennungsverarbeitung entwickelt sich Simon immer mehr zu einem Vollidioten. Die Hauptfigur ist gekennzeichnet von struktureller Unreife, die symptomatisch für den männlichen Teil der Generation zu sein scheint. Aus den Schilderungen ergibt sich das Bild eines komplett hilflosen Mannes, der, anstatt sein Leben in den Griff zu bekommen, seine ritualisierte Rauchsucht und erotische Hirngespinste pflegt.

Der im schnoddrigen Stil gehaltene Unterhaltungsroman trifft sicherlich nicht jedermanns Geschmack, zweifelsohne haben wir es aber mit einem Roman zu tun, der genrespezifische Merkmale des Single-Romans aufweist. Der Protagonist wurde mit typischen Single-Merkmalen ausgestattet. Simon lebt in einer Großstadt, wird demnächst dreißig, wurde vor einiger Zeit von seiner Freundin verlassen. Im Gegensatz zu Gregor Hamdorf, dem Protagonisten des Romans Die Frau fürs Leben von DANIEL BIELENSTEIN (2003), und im Gegensatz zu den meisten Darstellungen weiblicher Singles ist Simon, was die berufliche Laufbahn betrifft, ein unausgebildeter Versager. Die Erfahrung des Scheiterns zieht sich wie ein roter Faden durch das Leben des dargestellten Singlemannes, was eine deutliche Ich-Schwächung zur Folge hat. Das mangelnde Vertrauen auf eigene Gefühle und Lebensregeln und eine gewisse Orientierungslosigkeit machen sich in vielen Lebensbereichen bemerkbar. Aus diesem Grund wird nach externen Instanzen für Handlungsrichtlinien gesucht. Schon das Vorhaben, die Frau anzusprechen, von der er so sehr angetan ist, entwickelt sich zu einer unüberwindbaren Aufgabe, was Simon dazu veranlasst, Rat bei seiner Freundin Paula zu suchen, die über die Jahre zu einer Beziehungsexpertin schlechthin avanciert und in Beziehungsangelegenheiten deutlich versierter ist. Trotz der fast ausschließlich humoristischen Darstellung der Missverständnisse und Zwischenfälle, die die Liebe in Vollidiot mit sich bringt und die zu 
den genretypischen Merkmalen gehört, wird bei der Verbalisierung der Sehnsucht nach einer festen Bindung auf den manchmal gezwungenen Humor verzichtet. Eine Passage, in der das deutlich zum Ausdruck kommt, lautet wie folgt: „Ein plötzliches Gefühl von Leere und Einsamkeit wabert mir entgegen. Ich versuche auszuweichen, aber das Gefühl hat mich schon umhüllt.“ (JAUD 2012:13) An einer anderen Stelle liest man: „Mein ganzes, bisheriges Leben ist eine beschissene Pause [...]. Ich brauche eine Frau, die auf Start drückt, damit mein Leben weitergeht.“ (JAUD 2012:134)

\section{Fazit}

Analysiert man die Gegenwartsliteratur vor der Folie der veränderten Partnerschafts- und Lebensmodelle, stößt man auf Bilder brüchiger zwischenmenschlicher Beziehungen, bzw. es wird die Unmöglichkeit, feste Bindungen einzugehen, veranschaulicht. Trotz der offensichtlichen stilistischen und formalen Unterschiede zwischen den im Rahmen dieses Beitrags untersuchten Texten weisen diese eine Gemeinsamkeit auf, indem sie die Komplexität der zwischenmenschlichen Relationen thematisieren und gleichzeitig als eine kritische Bestandsaufnahme der modernen Gesellschaftslage aufgefasst werden können. Geht es in der Gegenwartsliteratur um Gefühle, so sind sie immer mit Desillusionierung verbunden. An dieser Stelle müsste man die Frage nach den möglichen Ursachen für diese Situation aufwerfen. Eine plausible Erklärung dafür liefert EVA ILLOUZ, die verkündet, dass Liebe ein durchaus entzaubertes Phänomen sei:

Die Liebe, wie wir sie in Westeuropa einige Jahrhunderte lang kannten, ist tot; sie wurde durch neue emotionale Formen ersetzt. Doch gleich dem Glanz längst erloschener Sterne scheint jene alte Form der Liebe fort und erfüllt uns noch immer mit Staunen über die Intensität ihres Leuchtens, lange nachdem sie in Wirklichkeit schon verschwunden ist. Die Liebe stand immer in einem gewissen Zusammenhang zur Sexualität, zur rationalen Wahl und zur Institution der Ehe. Der Inhalt und die Kombination dieser Elemente sind es, die sich in den zurückliegenden Jahrzehnten tiefgreifend verändert haben. ${ }^{11}$

Haben wir es mit einer Situation zu tun, in der Freiheit Geborgenheit schlägt und Individualität über Verbundenheit siegt? Aus der Lektüre der ausgewählten Werke ergibt sich das Bild einer ich-bezogenen Welt, die es nicht zulässt bzw. es enorm erschwert, Beziehungen zu anderen Menschen aufzubauen und

11 Illouz, EvA: Ist die Liebe tot? Aus dem Englischen von Michael Adrian: http://pdf.zeit.de/2013/25/eva-illouz-liebe-tot.pdf (30.06.2013). 
diese aufrechtzuerhalten. Das Leben in der flüchtigen Moderne, über das ZYGMUNT BAUMAN (2000 und 2005) schrieb, trägt dazu bei, dass die Menschen immer häufiger ein Problem damit haben, den einst eingeschlagenen Kurs beizubehalten, was sich natürlich negativ auf die Stabilität zwischenmenschlicher Beziehungen auswirkt. Diese Unbeständigkeit, die fast schon symptomatisch für die modernen, hyperaktiven sowie störungsanfälligen Gesellschaften ist, hat zur Folge, dass einige Lebens- bzw. Partnerschaftsmodelle als antiquiert gelten und deswegen durch gegenwärtige Formen des Zusammenlebens ersetzt werden.

\section{Literatur}

AdORJÁn, JohanNA (2004): Sie und ihr Maple Walnut. In: FAZ vom 2.05.2004. Online verfügbar: http://www.faz.net/aktuell/feuilleton/buecher/rezensionen/belletristik/sieund-ihr-maple-walnut-1163911.html (13.06.2013).

BAller, Susanne (2015): „Was in der ,Bravo’ geschieht, ist sexistisch und frauenfeindlich“: http://www.stern.de/familie/kinder/-sexistisch-und-frauenfeindlich---dr-sommer-geht-auf--bravo--los-6357054.html (18.09.2016).

Bauman, Zygmunt (2000): Liquid Modernity. Cambridge.

BAUMAN, ZYGmunt (2005): Liquid Life. Cambridge.

BECK, UlRICH (1983): Risikogesellschaft. Auf dem Weg in eine andere Moderne. Frankfurt/Main.

BeCK, UlRich / BeCK-GeRnsheim, ElisABeth (1990): Das ganz normale Chaos der Liebe. Frankfurt/Main.

Bielenstein, Daniel (2003): Die Frau fürs Leben. Frankfurt/Main.

Claus, BritTa (2012): Kein Leben zu zweit. Darstellungen des weiblichen Singledaseins in deutschsprachigen Romanen der Jahrtausendwende (1996-2006). Würzburg. Gansel, CARsten / HeRrmann, Elisabeth (2013): „,Gegenwart“ bedeutet die Zeitspanne einer Generation“ - Anmerkungen zum Versuch, Gegenwartsliteratur zu bestimmen. In: GANSEL, CARSTEN / HERRMANN, ElisABeth (eds.): Entwicklungen in der deutschsprachigen Literatur nach 1989. Göttingen, 7-22.

HöBel, Wolfgang (2006): Mausbär auf großer Fahrt. In: Der Spiegel, H. 30/2006:138.

Hradil, Stefan (1995): Die Single-Gesellschaft. München.

JaUd, TOMmy (2012): Vollidiot. Frankfurt/Main. 
ILlouZ, Eva (2012): Gefühle in Zeiten des Kapitalismus. Aus dem Englischen von Martin Hartmann. Frankfurt/Main.

ILlouz, Eva (2013): Ist die Liebe tot? Aus dem Englischen von Michael Adrian. In: Die Zeit vom 20.06.2013. Online verfügbar: http://pdf.zeit.de/2013/25/eva-illouz-liebetot.pdf (30.06.2013).

KLINENBERG, ERIC (2012): Going Solo. The Extraordinary Rise and Surprising Appeal of Living Alone. New York.

KŁopotowska, Justyna (2013): Weiblichkeit und Identität im Wandel. Angelika Reitzers poetische Auseinandersetzungen mit der Wirklichkeit. In: DRYNDA, JOANNA / Wimmer, MARTA (eds.): Neue Stimmen aus Österreich. 11 Einblicke in die Literatur der Jahrtausendwende. Frankfurt/Main, 111-120.

KnAUP, ANNA KATHARINA (2015): Der Männerroman: Ein neues Genre der deutschsprachigen Gegenwartsliteratur. Bielefeld.

KÜPPER, BEATE (2000): Sind Singles anders als die anderen? Ein Vergleich von Singles und Paaren: http://www-brs.ub.ruhr-uni-bochum.de/netahtml/HSS/Diss/KuepperBeate/diss.pdf, (15.06.2013).

Mensing, Kolja (2004): Marlene Streeruwitz: Jessica, 30. Die Rache des Schokobechers. In: Frankfurter Allgemeine Zeitung vom 12.05.2004, Nr. 110.

Metz, Christian (2012): Die Narratologie der Liebe: Achim von Arnims „Gräfin Dolores “. Berlin / Boston.

PEUCKERT, RÜDIGER (2012): Familienformen im sozialen Wandel. Wiesbaden.

Pohl, IsABella (2012): Gemeinsam sein und doch getrennt. In: Der Standard vom 25.08.2012. Online verfügbar: http://derstandard.at/1282273499151/Gemeinsam-seinund-doch-getrennt-leben (29.06.2013).

Polt-Heinzl, Evelyne: Krisenstoff oder Was erzählt die österreichische Literatur über unsere Befindlichkeit im neuen Jahrtausend. In: DRYNDA, JOANNA / WIMMER, MARTA (eds.): Neue Stimmen aus Österreich. 11 Einblicke in die Literatur der Jahrtausendwende. Frankfurt/Main, 11-24.

Reitzer, AngeliKa (2010): unter uns. St. Pölten / Salzburg.

REITZER, ANGELIKA im Interview mit Bettina Führer: http://www.buecher.at/show_content2.php?s2id=452 (25.06.2013).

StreEruWitz, MARLENE (1997): Lisa's Liebe. Roman in 3 Folgen. Frankfurt/Main.

STREERUWITZ, MARLENE (2004): Jessica 30. Frankfurt/Main.

STRIGL, DANiEla (2010): Wenn man das Leben nur noch mitmacht. In: Falter: Buchbeilage 39/2010 vom 29.9.2010. 
Tyrell, Hartmann (1979): Familie und gesellschaftliche Differenzierung. In: Pross, HELGE (ed.), Familie - wohin? Leistungen, Leistungsdefizite und Leistungswandlungen der Familien in hochindustrialisierten Gesellschaften. Reinbek, 13-77.

Wagner, Michael / Franzmann, Gabriele / Stauder, Johannes (2001): Neue Befunde zur Pluralität der Lebensformen. In: Zeitschrift für Familienforschung 13, Heft 3/2001:52-73.

ZinTZEn, Christiane (2005): System Streeruwitz: Morire in Levitate. In: Literaturen 3:68-71. 